\title{
Automatização Total do Diagnóstico de Malária - Fase 1: Teste de Viabilidade - Modelo Classificatório Fuzzy
}

\author{
Yuri Vasconcelos de Almeida Sá \\ UNESP Campus Sorocaba - Mestrando - Ciências Ambientais \\ Sorocaba/SP (e-mail:yuri@sa2.com.br).
}

\begin{abstract}
This work offers a Fuzzy Inference System to classify images from microscope slides for Malaria diagnosis. This article formalizes the theoretical basis for the creation of an autonomous, offline Malaria diagnosis device with a positive result superior to the first works in the field, even in its early development states (proof of concept).
\end{abstract}

Resumo: Este trabalho tem como objetivo propor um sistema fuzzy para classificar imagens oriundas de microscopia para o diagnóstico da Malária . Este artigo formaliza a base teórica para a criação de um dispositivo autônomo, offline, de diagnóstico da doença com um acerto superior aos primeiros trabalhos da área, mesmo em seu estágio inicial (prova de conceito).

Keywords: Fuzzy Logic, Malaria Diagnosis, Machine Learning, Diagnosis Automation, Bioinformatics.

Palavras-chaves: Lógica Fuzzy, Diagnóstico da Malária, Aprendizado de máquina, Automação de diagnóstico, Bioinformática

\section{INTRODUÇÃO}

A malária é um problema de saúde global. A doença está em crescimento em 16 países (CNN, 2018). Um diagnóstico rápido é chave para seu controle. O método mais confiável e aceito é um técnico/médico analisar uma amostra de sangue (gota e esfregaço) através de microscopia com coloração (Brasil, 2018).

Por se tratar de uma doença cujo diagnóstico é visual e depende de um profissional qualificado e experiente para que os resultados sejam minimamente confiáveis, os modelos computacionais modernos começaram a criar soluções teóricas para seu diagnóstico automatizado. Este problema se tornou um exemplo de processamento de imagens digitais, com inúmeros trabalhos publicados nos últimos anos sobre o tema.

Porém, na literatura relevante, não existe um trabalho que busque o diagnóstico real a partir de amostras - e com equipamentos de baixo custo (processamento digital limitado, offline). O mais próximo que chegou-se de automatização real, em campo, foi a aplicação descrita pelo Departamento de Saúde Norte-Americano (NIH, 2018), para Smartphones Android, porém sua utilização ainda é manual e depende de vários recursos para sua operação (microscópio, operador, celular, etc.).

A análise utilizada aqui foi feita puramente através das características da imagem e seus atributos estatísticos. Este primeiro passo para que o modelo seja criado dentro das capacidades de plataformas digitais sem co-processador numérico, ex.: Arduino, NodeMCU, etc.

Este trabalho descreve o primeiro passo para a criação de um equipamento de baixo custo que, a partir da amostra de sangue, o diagnóstico seja apresentado com o mínimo de confiabilidade.

\section{FUNDAMENTAÇÃO TEÓRICA}

Devido ao advento da redução de custo das CPUs e GPUs experimentado nos últimos anos, inúmeros modelos e bibliotecas para processamento de enormes quantidades de dados foram criados, possibilitando um grande desenvolvimento de métodos e técnicas de processamento de imagem. Os modelos recentes são capazes de processar volumes muito grandes de dados e manipulá-los de maneiras inimagináveis anteriormente.

Todos os últimos modelos desenvolvidos utilizam uma ou mais formas de redes neurais, uma técnica que aumenta demais a complexidade do modelo e o custo de processamento (CPU). Esses modelos exigem treinamento e uma vasta coleção de bibliotecas para funcionar, sendo que o modelo que, até hoje, tem a maior precisão (teórica), se utiliza de Redes Neurais Convolucionais com até 16 camadas para que sejam efetivamente utilizados e, mesmo assim, se valeram de recortes e subutilização do corpo amostral devido ao seu alto custo computacional. A tendência a melhores resultados é a utilização deste tipo de redes neurais como 
melhor caminho para a excelência do resultado. (Rajaraman, 2018)

Como referência, a Tabela 1 exibe os trabalhos sobre classificação de amostras de sangue contaminadas com o plasmódio da malária na literatura recente.

\begin{tabular}{l|l} 
Método & Precisão \\
\hline Rajaraman et al. (2018) & 0.959 \\
\hline Gopakumar et al. (2018) & 0.977 \\
\hline $\begin{array}{l}\text { Bibin, Nair \& Punitha } \\
\text { (2017) }\end{array}$ & 0.963 \\
\hline Dong et al. (2017) & 0.981 \\
\hline Liang et al. (2017) & 0.973 \\
\hline Das et al. (2013) & 0.840 \\
\hline Proposição deste trabalho & 0.769 \\
\hline Ross et al. (2006) & 0.730
\end{tabular}

Tabela 1 - Evolução da precisão dos modelos de classificação e diagnóstico da Malária

\section{METODOLOGIA}

\subsection{Aquisição dos dados}

Os dados foram obtidos através do Instituto Nacional de Saúde estadunidense (NIH, 2018), no qual já existe um dataset contendo 26.558 imagens, classificadas como sadias e infectadas. As imagens contidas possuem 3 canais RGB e resoluções de 100x100 a 299x299 pixels (Rajaraman, 2018).

Para que estas imagens possam ser utilizadas seguindo um modelo de visão computacional, todas foram importadas no software estatístico R e trabalhadas como uma Matriz.

$\mathrm{O}$ ajuste da entrada (Feature Engineering) é o processo de normalização, adequação, agrupamento, combinação e decomposição das variáveis, para que o modelo preditivo/classificatório (GUYON, 2003) as compreenda melhor .

De posse da imagem, houve a separação de cada canal e a remoção de pixels mortos (Valor igual $0 \mathrm{em}$ todos os canais). Então, para cada canal, foi feita a média dos valores de cada pixel (variando de 0 a 255), que busca identificar marcações que são escuras, baixando o valor geral dos pixels (Figura 1); o valor mínimo de um pixel individual na imagem que busca verificar se possui alguma região muito escura, por fim; o número de pixels cujo valor está abaixo de $25 \%$ do máximo encontrado no canal, que busca encontrar concentrações de marcações escuras na imagem.
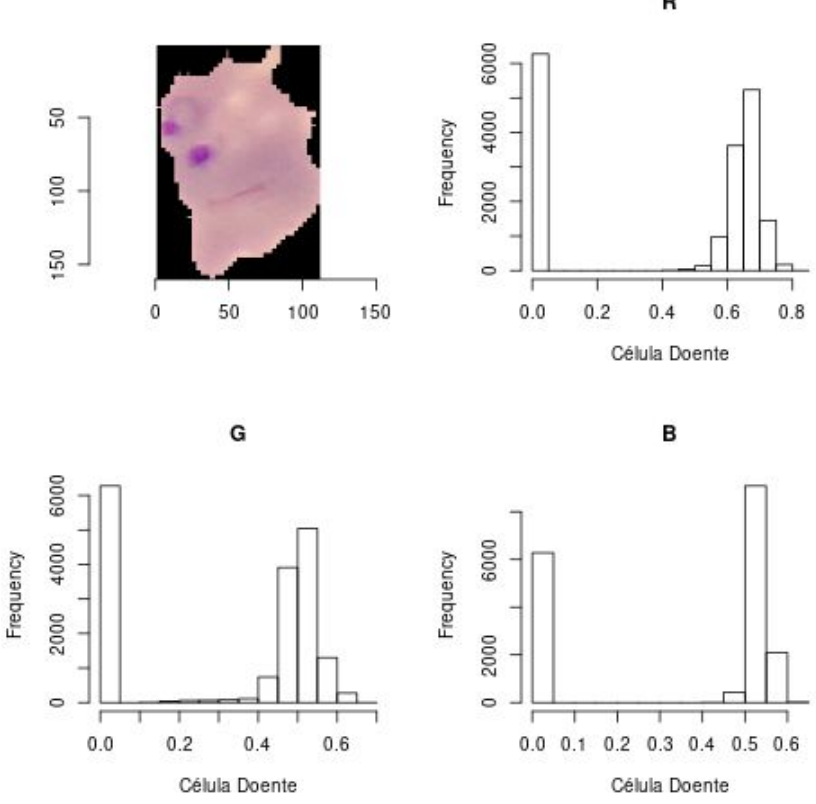

Figura 1 - Uma amostra infectada e os histogramas de cada canal, é importante observar a redução da média devido aos pontos escuros na imagem.
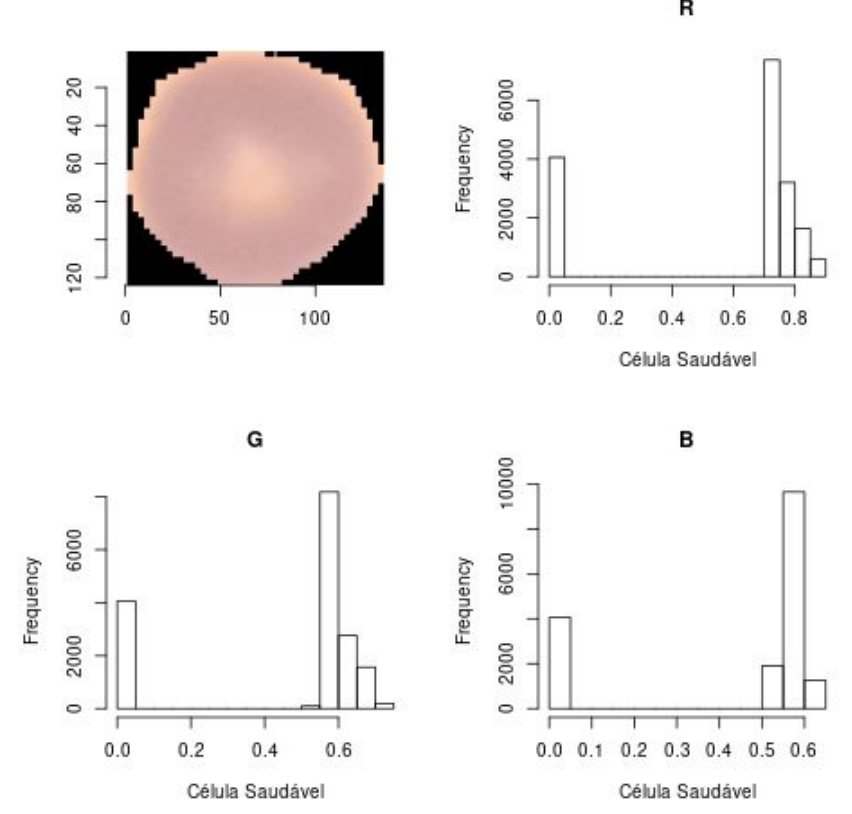

Figura 2 - Uma amostra saudável é mais uniforme e tende a ser mais clara, por isso existe uma elevação e concentração dos pixels mais claros. 
O total de features ficou em 6, com mais uma coluna de resultado $(0 ; 1)$ para infectado ou não.

Cada imagem gera então uma assinatura única, que pode ser descrita em 7 colunas.

\section{2 - A Lógica Fuzzy}

A lógica fuzzy busca determinar um número que descreve um conjunto elevado de variáveis incertas e vagas associando intervalos a variáveis linguísticas, flexibilizando a aplicação da lógica clássica, estendendo e facilitando seu uso em condições multivariadas e multidimensionais; agregando valores a variáveis além do conceito binário $[0,1]$ de modo que sua implementação possibilita o tratamento de variáveis que antes fugiam ao controle (SHAW; SIMÕES, 1999).

A primeira ocorrência da expressão "lógica fuzzy" (nebulosa) para ser utilizada com base na teoria de conjuntos fuzzy foi usada no artigo Fuzzy Sets (ZADEH, 1965).

A característica de lidar com a ambiguidade da informação e a incerteza do mundo real fez com que este raciocínio fosse aplicado em pesquisas de diversas áreas, tais como sistemas de controle e inteligência artificial devido sua capacidade de imitar o raciocínio humano, que considera verdades parciais ou graus de verdade. (GIGCH; PIPINO, 1980)

Na lógica fuzzy, as variáveis são associadas a termos que definem seu pertencimento e as operações são feitas a partir da linguagem natural, cujo maior benefício é a codificação de conhecimentos inexatos, se aproximando de um modelo cognitivo, característicos da mente humana (RUHOFF et al., 2005).

\section{3 - A Lógica Fuzzy na visão computacional}

Devido ao volume enorme de dados que uma única imagem nos trás (uma imagem VGA (640x480px) contém 307200 por canal, se for colorida 921600 pixels no total), cada imagem representa um problema de dados estatisticamente muito rico e, portanto, passível de utilização de diferentes técnicas de Inteligência Artificial.

A lógica fuzzy, com gradientes de verdade e pertencimento apresenta traços diferentes das técnicas já utilizadas pois diferentemente da evolução que analisamos nos algoritmos de classificação de imagens, como a própria $C N N$ (Convolutional Neural Networks), a lógica fuzzy não requer treino (otimização) ou grande custo computacional.

\subsection{A separação do dataset com base no resultado}

\subsubsection{Definindo resultado}

O dataset descrito (NIH, 2018), já é classificado por um técnico em microscopia, que contém imagens de lâminas segmentadas de 150 pacientes infectados pelo plasmódio da malária e 50 pacientes saudáveis, apesar do número de pacientes ser diferentes, o número de lâminas infectadas e saudáveis é o mesmo.

\subsubsection{Separando os resultados em datasets distintos}

O sistema estudado é um classificador binário, portanto, o melhor método para atingir tal resultado utilizando lógica Fuzzy é avaliar a probabilidade de cada resultado possível (0 - Células sadias, 1 - células doente (com plasmódio da malária)).

Cada dataset terá um sistema Fuzzy completo individualizado (entrada, funções de pertencimento, regras e saída próprios), e que o resultado de cada um seja a probabilidade de cada um acontecer, calculada pelo FIS.

\subsubsection{Criação de sistemas Fuzzy (FIS) paralelos}

Para que a classificação seja feita, dois sistemas fuzzy (FIS) são criados, e calculados independentemente, utilizando as mesmas variáveis, porém separadas pelo resultado. Conforme diagrama da Figura 3.

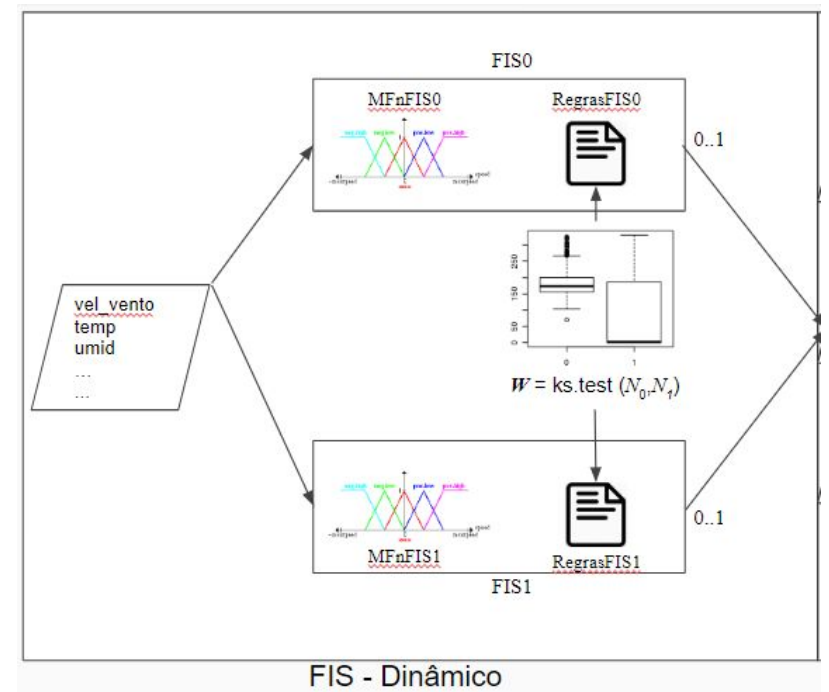

Figura 3 - Descreve o funcionamento dos dois sistemas fuzzy paralelos.

Ao final, cada consulta e execução dos sistemas fuzzy (um sistema para o resultado positivo e outro para o resultado negativo) devem retornar dois resultados independentes e simultâneos (não complementares). Onde o maior prevalece, o resultado obtido de cada sistema é estipulado de 0 a 1 , e o maior é considerado o resultado.

Toda a implementação fuzzy foi feita no pacote FuzzyR (GARIBALDI, J, 2017), que contém todas as funções básicas do cálculo fuzzy baseado em regras e uma interface muito similar ao padrão da indústria, com arquivos de extensão .fis e importação das regras via matriz, tornando a experimentação rápida e prototipagem muito mais rápida $\mathrm{e}$ eficiente. 


\subsection{A formação das funções de pertinência}

O sistema deve ser o mais dinâmico possível para que atenda o maior número de datasets e variáveis possível, para tanto, as funções de pertinência devem ser dinâmicas e auto-calculadas.

Dentre os muitos métodos possíveis, os mais populares utilizam redes neurais e algoritmos genéticos para definir as funções de pertinência, embora estejam bem estabelecidas na literatura, consomem muito tempo e recursos computacionais (ASANKA, 2017).

O método utilizado no trabalho, descrito na Figura 4 abaixo, define os limites através dos valores obtidos nos box-plots das colunas (Quartis e mediana de cada feature), criando 3 estágios de probabilidade sendo a mediana a maior probabilidade e quanto mais afastado, menor. Os dois conjuntos mais externos (distantes da mediana) tem a pertinência BAIXA, os dois intermediários (mais próximos da mediana) tem a pertinência MÉDIA e o conjunto (somente um) da média tem a pertinência alta. Os prováveis outliers são excluídos na camada de alimentação do algoritmo para reduzir o ruído da amostra.

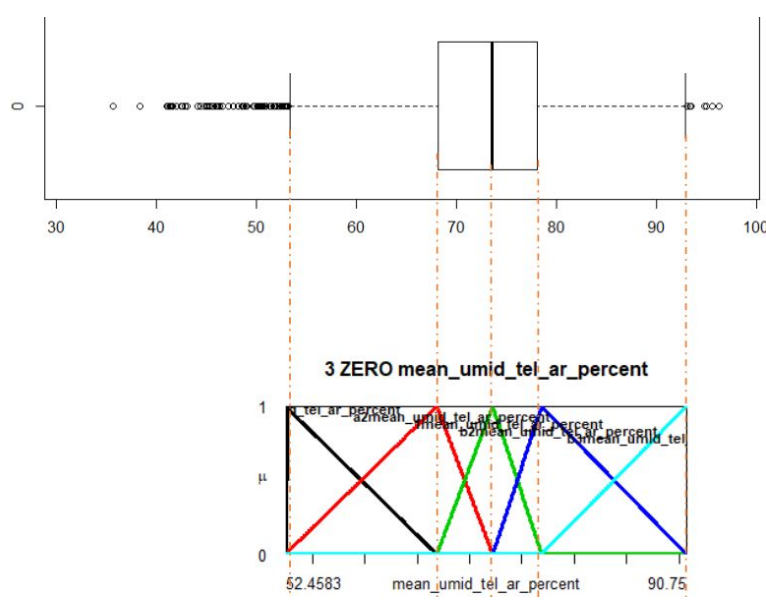

Figura 4 - Boxplots gerando funções de pertinência

Os dados obtidos a partir das primeiras 100 imagens de cada dataset (infectados e saudáveis) e podem ser gravadas e transferidas para um modelo de produção. Embora o próprio sistema, quando concluído, possa facilmente criar suas próprias funções de pertinência, o aumento do corpo amostral não tem relação com um aumento da precisão do modelo.

\section{7 - Criação das regras}

$\mathrm{O}$ modelo de Mamdani, utiliza-se de conjuntos que recebem uma atribuição linguística que são associadas por uma base de regras (Zadeh, 1965), cada regra deve associar uma entrada com uma saída..
Diferentemente de outros métodos de Inteligência Artificial /Machine Learning que dependem de aprendizado (otimização), o Sistema Fuzzy não requer treino, porém sofre do tamanho da lista de regras.

Uma estratégia foi divisada para superar este obstáculo, que mesmo alterando o resultado numérico do sistema Fuzzy, ainda dá a direção correta do cálculo, foram feitas então as regras incompletas do sistema, não relacionando as variáveis de entrada entre si, cada variável de entrada somente tem relação com o resultado.

Em uma relação com a Estatística, isso torna o sistema Fuzzy Univariado, sendo que o resultado final é um acumulado de todas as variáveis calculadas individualmente.

Para atingir essa tabela, o sistema cria um modelo preenchido com " 0 " (Regra vazia) e escreve em cima de cada regra somente, a partir de um modelo.

Utilizando este método, invés de $\mathrm{E}^{\wedge} \mathrm{V}$ (Entrada elevada a variações), convertemos o número de regras para $\mathrm{E} * \mathrm{~V}$ (Entrada multiplicada pelas variações). Porém exclui-se a influência de uma variável sobre a outra e suas correlações.

\section{8 - A aplicação de pesos}

O conceito de regras limitadas empregado neste trabalho facilita a aplicação de pesos a cada variável, portanto, é possível criar pesos diferentes para cada entrada no sistema, fazendo com que o resultado penda para as variáveis com maior peso.

O peso das regras de cada variável é calculado a partir da diferença da distribuição normal entre a mesma variável no dataset com resultado 0 e o dataset com resultado 1 , utilizando o Teste Kolmogorov-Smirnov.

Para uma maior otimização dos pesos (maximização dos resultados), todos os pesos foram colocados em uma matriz e então normalizados, ou seja, a maior diferença gera um peso de valor 1 , e a menor de valor 0 (o que não é calculado no sistema Fuzzy resultante), conforme exemplificado na Figura 5 abaixo.

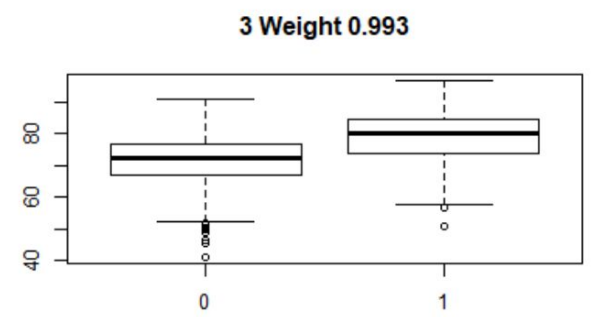

Figura 5 - Boxplots representando a distribuição normal da mesma variável nos datasets 0 e 1, quanto mais diferente, maior o peso (weight)

\section{9 - Funções de resultado}


O resultado dos dois sistemas é compilado em uma tabela junto ao resultado classificado do dataset (resultado original) e compila o cálculo em uma Matriz confusão.

\section{4 - APLICAÇÃO E RESULTADOS}

Devido à natureza homogênea do objeto (análise de imagens não é cíclica ou acontece em períodos e não existe autocorrelação como em séries temporais), os experimentos e seus resultados também foram desenhados desconsiderando períodos.

Uma vez que este trabalho é baseado em conhecimento de domínio, a escolha das variáveis também foi feita utilizando este raciocínio. A matriz confusão e suas características principais estão listadas abaixo:

\begin{tabular}{|c|c|c|c|}
\hline $\operatorname{Ref}$ & Eerence & & \\
\hline Prediction & 0 & 1 & \\
\hline 0 & 10520 & 3722 & \\
\hline 1 & 3158 & 12256 & \\
\hline & $A C C$ & curacy & $: 0.7680$ \\
\hline & Sen & sivity & $: 0.7671$ \\
\hline & Specif & ficity & $: 0.7691$ \\
\hline & Pos Pred & Value: & $: 0.7951$ \\
\hline & Jeg Pred & Value: & $: 0.7387$ \\
\hline & $\mathrm{F} 1$ & Score & $: 0.7808$ \\
\hline
\end{tabular}

\section{CONCLUSÕES}

Diante do resultado, fica claro que o sistema proposto tem capacidade de classificar células doentes com um mínimo de informação.

É necessário, porém, testes e ensaios para que mais variáveis sejam criadas e auto-selecionadas, com base estatística.

O sistema e seu script $R$, como está posto também apresenta um consumo muito baixo de recursos computacionais, tornando sua utilização e teste extremamente ágeis em um computador pessoal intermediário.

Embora o modelo esteja muito aquém de experimentos recentes envolvendo tecnologias avançadas $(C N N)$, ainda assim apresenta acertos de $79.51 \%$ no resultado positivo, tornando-o qualificado para desenvolvimentos futuros. Sendo a próxima fase da pesquisa o ensaio do sistema embarcado em plataformas digitais.
Alves, Aj. EFLL (Embedded Fuzzy Logic Library) is a standard library for Embedded Systems. Disponível em: <https://github.com/zerokol/eFLL>. Acesso em: 20 set. 2018 .

Asanka, PPG Dinesh; PERERA, Amal Shehan. DEFINING FUZZY MEMBERSHIP FUNCTION USING BOX PLOT. 2017.

Bibin, Dhanya, Madhu S. Nair, and P. Punitha. "Malaria parasite detection from peripheral blood smear images using deep belief networks." IEEE Access 5 (2017): 9099-9108.

Brasil. Ministério da Saúde. . Manual de Diagnóstico Laboratorial da Malária. 2005. Disponível em: $<$ http://bvsms.saude.gov.br/bvs/publicacoes/malaria _diag_manual_final.pdf $>$. Acesso em: 01 out. 2016.

CNN (Us) (Ed.). Malaria on the rise in more than 13 countries, experts warn. 2018. Disponível em: $<$ https://www.cnn.com/2018/11/19/health/malaria-in crease-global-report-2018-intl/index.html>. Acesso em: 19 nov. 2018.

Das, Dev Kumar, et al. "Machine learning approach for automated screening of malaria parasite using light microscopic images." Micron 45 (2013): 97-106.

Dong, Yuhang, et al. "Evaluations of deep convolutional neural networks for automatic identification of malaria infected cells." 2017 IEEE EMBS International Conference on Biomedical \& Health Informatics (BHI). IEEE, 2017.

Estados Unidos. NIH. (Org.). Malaria Datasets. 2018. Disponível em:

$<$ https://ceb.nlm.nih.gov/repositories/malaria-dataset s/>. Acesso em: 01 out. 2018.

Garibaldi, J, CHAO C. FuzzyR: Fuzzy Logic Toolkit, 2017

for $\quad$ R. $\quad$ R package version
https://CRAN.R-project.org/package=FuzzyR

Guyon, Isabelle; ELISSEEFF, André. An introduction to variable and feature selection. Journal of machine learning research, v. 3, n. Mar, p. 1157-1182, 2003.

Gigch, J. ; Pipino, L. Form Absolute to Probable to Fuzzy in Decision Making. Kybernetes, v. 19, p. 433-461, 1980 .

Gopakumar, Gopalakrishna Pillai, et al. "Convolutional neural network-based malaria diagnosis from focus stack of blood smear images acquired using custom-built slide scanner." Journal of biophotonics 11.3 (2018): e201700003. 
Kuhn, M. caret: Classification and Regression Training. R package version 5.16-24, 2018.

Lander, J. et al.useful: A Collection of Handy, Useful Functions. R package version 1.2.6, 2018

Liang et al., "CNN-based image analysis for malaria diagnosis," 2016 IEEE International Conference on Bioinformatics and Biomedicine (BIBM), Shenzhen, 2016, pp. 493-496.

Rajaraman, Sivaramakrishnan, et al. "Pre-trained convolutional neural networks as feature extractors toward improved malaria parasite detection in thin blood smear images." PeerJ 6 (2018): e4568.

Ross, Nicholas E., et al. "Automated image processing method for the diagnosis and classification of malaria on thin blood smears." Medical and Biological Engineering and Computing 44.5 (2006): 427-436.

Zadeh, L. A. Fuzzy sets. Information and Control, New York, v. 8, p. 338- 353, 1965. 